\title{
Richter Hernia As a Complication of Ventriculoperitoneal Shunt Placement
}

\author{
Quratulain Sabih, MD, Timothy Perez, MD, MPH, FACS \\ Department of Surgery, University of New Mexico School of Medicine, Albuquerque, New Mexico, USA (all authors).
}

\begin{abstract}
Richter hernia results from herniation of the antimesenteric wall of the bowel without compromising its lumen. Insidious onset and misleading symptoms lead to delay in diagnosis and a high mortality. We report a case of Richter hernia, after ventriculoperitoneal shunt placement which was later reduced and the defect closed laparoscopically.
\end{abstract}

Key Words: Richter hernia, Complication, Laparoscopic management.

Citation Sabih Q, Perez T. Richter hernia as a complication of ventriculoperitoneal shunt placement CRSLS e2014.00129. DOI: 10.4293/CRSLS.2014.00129.

Copyright (C) 2014 SLS This is an open-access article distributed under the terms of the Creative Commons Attribution-Noncommercial-ShareAlike 3.0 Unported license, which permits unrestricted noncommercial use, distribution, and reproduction in any medium, provided the original author and source are credited.

Address correspondence to: Timothy Perez, Department of Surgery, UNM School of Medicine, University of New Mexico, Albuquerque, NM 87131-0001. E-mail: TPerez@salud.unm.edu.

\section{CASE HISTORY}

A 33-year-old man with hydrocephalus requiring multiple ventriculoperitoneal (VP) shunt revisions presented with persistent vomiting for 3 days after his most recent shunt revision. This revision involved placement of the peritoneal portion of the VP shunt via a mini-laparotomy. He had been discharged home within 24 hours of the surgery. He presented to the emergency department because of his unremitting symptoms and progressive lethargy. On examination he appeared lethargic and dehydrated. His abdomen was soft but minimally distended, no masses were palpable, and no tenderness could be elicited. A nasogastric tube was placed, with an immediate return of $1 \mathrm{~L}$ of coffee ground appearing fluid.

A shunt series reported "mild kinking" of the tube, however demonstrated abnormal bowel gas patterns consistent with small bowel obstruction. Subsequent computed tomography scan of the abdomen demonstrated "mild to moderate gaseous and fluid distention of multiple loops of small bowel with a focal transition point near the ventriculoperitoneal shunt catheter entrance likely due to an adhesion" (Figure 1). Pneumatosis of the duodenum and proximal bowel and scattered foci of possible portal venous gas were also noted. There was not an obvious herniation of the small bowel.

Because of the concern for a mechanical small bowel obstruction due to the VP shunt insertion, the patient was taken to the operating room urgently. Diagnostic laparoscopy was performed, and a loop of small bowel was seen to be herniated through the anterior abdominal wall at the site of the VP shunt surgical site (Figure 2). The bowel appeared viable and was reducible with minimal traction.

A significant fascial defect was noted around the VP shunt tube (Figure 3), which was closed with interrupted nonabsorbable sutures using the laparoscopic technique. The peritoneal end of the shunt was observed to remain functional after this repair. The bowel that had initially appeared congested rapidly returned to normal appearance and peristalsis. The pneumatosis and small amount of portal venous gas were felt to be benign findings (Figure 4).

The patient made an uneventful recovery and was discharged on postoperative day 2 tolerating oral intake. His follow-up visit 2 weeks after discharge was unremarkable for complications. Since then, he has been seen by his primary care physician, with no complaints related to his surgery.

\section{DISCUSSION}

Richter hernias comprise $10 \%$ of strangulated hernias. This entity was first described in 1598 by Fabricius Hildanus, and again in 1887 by Sir Frederick Treves, whose observations and interpretation are the basis of our knowledge of this interesting entity. Because only a portion of the bowel circumference is involved in Richter hernia, signs of 
Richter Hernia As a Complication of Ventriculoperitoneal Shunt Placement, Sabih Q et al.

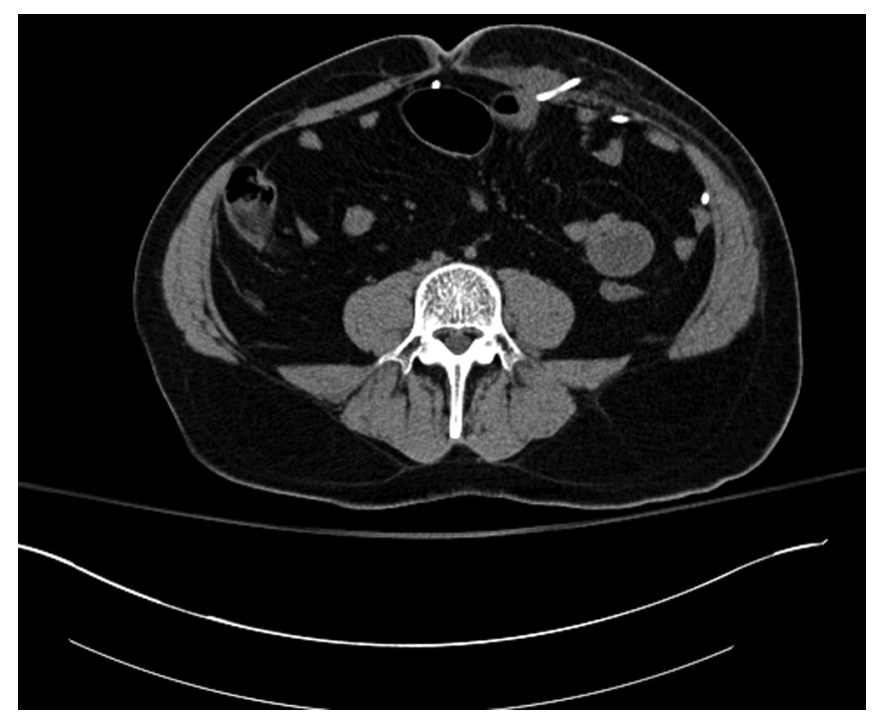

Figure 1. Computed tomography scan of the abdomen. The arrow marks the proximity of the small bowel to the site of VP shunt entrance.

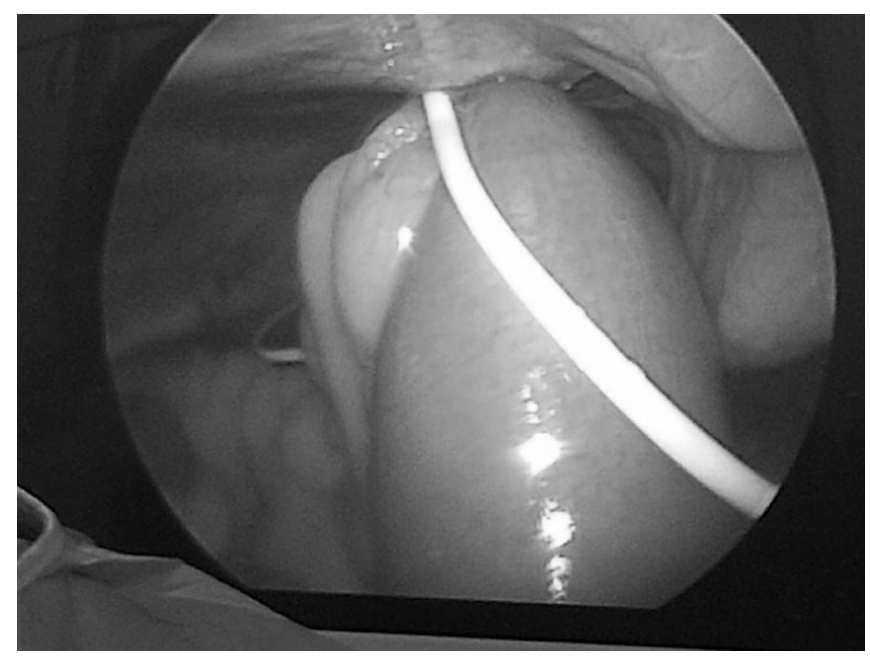

Figure 2. Laparoscopic view of the small bowel loop herniating through the VP shunt surgical site.

obstruction can be absent, but the involved part of the bowel can become rapidly gangrenous, with a resultant high mortality rate. ${ }^{1,2,3}$ Fortunately, mortality has significantly decreased from $62.2 \%$ in the first observed cases about 400 years ago to $17 \%$ as observed by Steinke and Zellweger's series in 2000. ${ }^{4}$ Compared with other hernias, Richter hernias have greater preoperative delay, rate of bowel resection, and complication and mortality rates. ${ }^{1}$ Common sites are the femoral and inguinal rings and locations of incisional trauma. ${ }^{3}$ Richter hernia is a rare complication of laparoscopic surgery, ${ }^{5}$ with trocar sites

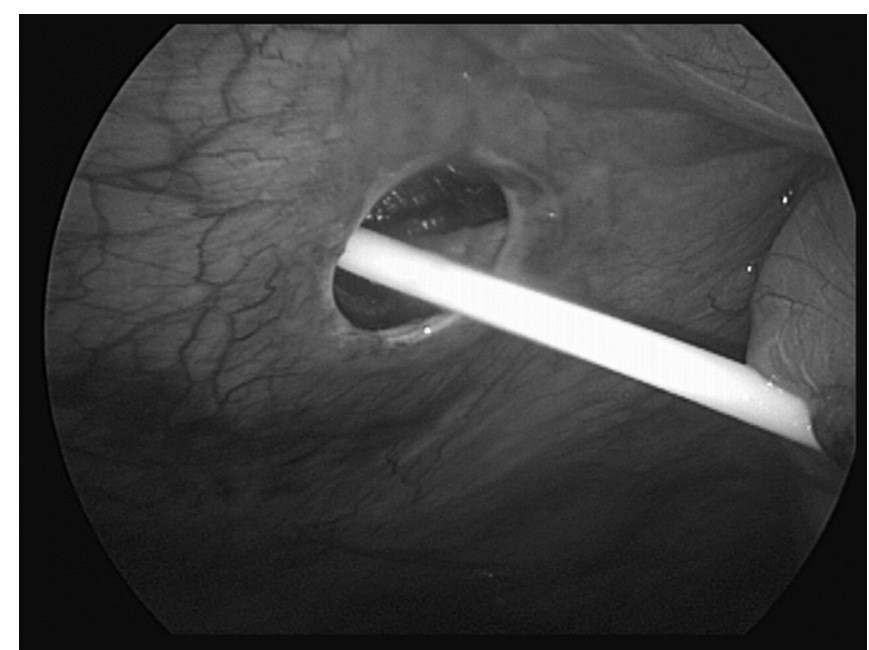

Figure 3. Fascial defect after reduction of Richter hernia.

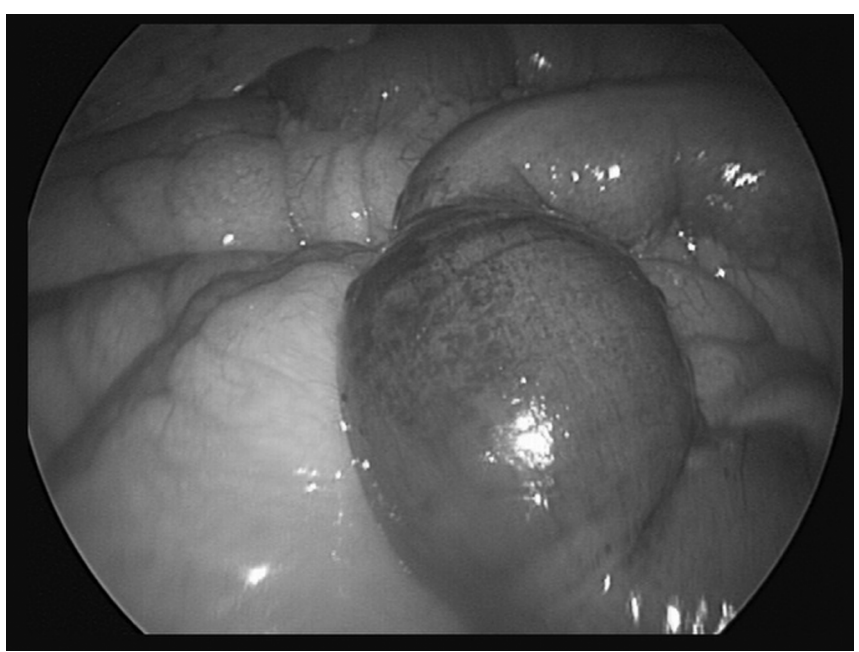

Figure 4. The herniated segment of bowel post reduction.

being an area of potential susceptibility. ${ }^{2,5}$ It has been described at a drain insertion site ${ }^{6}$ and has been reported at a trocar site into a deep preperitoneal space despite fascial closure. ${ }^{7}$

No reports of a Richter hernia after a VP shunt surgery have been published. Instances of herniation associated with drain or trocar sites have occurred only after the removal of the drain or trocar. A number of inferences can be made given the unusual nature of this case. Although incarceration of the small bowel was suspected preoperatively in our patient, a Richter hernia was discovered only on surgery:

1. The hernia occurred alongside a functioning VP shunt. This is significant in that the presence of a tube in situ might 
reduce the index of suspicion. Our case highlights that even if an abdominal tube is in place, this does not exclude the chances of this rare, potentially fatal, occurrence.

2. A prospective study by Roth et al of 211 patients over a 5-year period ${ }^{9}$ indicated a lower malfunction rate of laparoscopic VP shunt placements, and their discussion supported laparoscopy as a safer alternative in patients with histories of significant abdominal operations, peritonitis, and multiple revisions. Their argument against a laparotomy is to prevent adhesions. Although a larger trochar incision would act for all intents and purposes as a minilaparotomy, our findings are consistent with the literature. Laparoscopy in this case may have been safer, with a lower chance of adhesions developing, but given the patient's previous surgeries, this would have been technically more challenging.

\section{CONCLUSIONS}

Richter hernia is a rare and potentially grave complication seen where small fascial defects secondary, i.e previous drain sites, to laparoscopic trocar sites or small laparotomy incisions are present.

Our case highlights how a laparoscopic approach can be a useful adjunct to the management of VP shunt-related complications including Richter's hernia.

\section{References:}

1. Kadirov S, Sayfan J, Friedman S, Orda R. Richter's hernia-a surgical pitfall. J Am Coll Surg. 1996;182:60-62.
2. Williams MD, Flowers SS, Fenoglio ME, Brown TR. Richter hernia: a rare complication of laparoscopy. Surg Laparosc Endosc. 1995;5:419-421.

3. Skandalakis PN, Zoras O, Skandalakis JE, Mirilas P. Richter hernia: surgical anatomy and technique of repair. Am Surg. 2006;72:180-184.

4. Steinke W, Zellweger R. Richter's hernia and Sir Frederick Treves: an original clinical experience, review, and historical overview. Ann Surg. 2000;232:710-718.

5. Bendsen AK, Bauer T, Johansen TP. [Richter hernia in trocar site after laparoscopic herniotomy]. Ugeskr Laeger. 1995;157: 6438-6439.

6. Iwase K, Higaki J, Mikata S, et al. Ileal perforation due to a Richter hernia at the drain insertion site following an operation for idiopathic rectal perforation: report of a case. Surg Today. 2000;30:66-68.

7. Matthews BD, Heniford BT, Sing RF. Preperitoneal Richter hernia after a laparoscopic gastric bypass. Surg Laparosc Endosc Percutan Tech. 2001;11:47-49.

8. Boughey JC, Nottingham JM, Walls AC. Richter's hernia in the laparoscopic era: four case reports and review of the literature. Surg Laparosc Endosc Percutan Tech. 2003;13:55-58.

9. Roth J, Sagie B, Szold A, Elran H. Laparoscopic versus nonlaparoscopic-assisted ventriculoperitoneal shunt placement in adults. A retrospective analysis. Surg Neurol. 2007;68:177-184; discussion 184. 